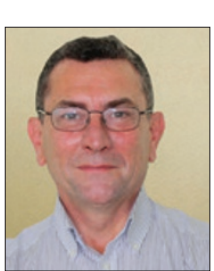

\section{IN THIS ISSUE}

In this issue

Professor Francis

Hughes and fellow

authors explore the

prevalence of peri-

odontal disease in a

Romano-British population c.200-

400 AD (pages 459-465).

Professor Hughes told the $B D J$ :

'We carried out this work for two reasons - firstly because of a long-standing interest in aetiological factors for periodontitis, and secondly through serendipity. I was talking with my friends and colleagues, Drs Ginny Kingsmill and Helen Liversidge, at Barts \&t The London, and somehow we got on to discussing these collections of skeletal remains from ancient burial grounds. It seemed like a very interesting and unusual way to look at the natural history of periodontal disease and with Helen's help we were able to set up the study with the Natural History Museum.

'We seem to have far too little time to simply sit down and talk about ideas, but often this can be a most fruitful way of spending some time. Having spent much of my research career doing esoteric things in cell culture laboratories, this study was simply fascinating. I think this was partly because of the feeling of very real human connections back to a time we knew little about. My colleague and first author Tuija Murray did most of the hard work and data collection but it was always completely engaging for me too.

The study opens up some interesting further questions, including looking at other ancient populations, and carrying out more detailed analyses such as genetic testing and microbial DNA identification using modern forensic techniques.'

Professor Hughes, who is professor of periodontology at King's College London Dental Institute, went into dentistry for reasons that he thinks are common for many, including a great interest in biological science, and a desire to do something "practical' and with people.

'It is also interesting to think of my further aspiration of wanting to work for myself, given that I have finished up working as a small cog in the largest dental hospital in Europe!'

\title{
PHOTO CHALLENGE
}

Dr Archana Singh, a professor in periodontology and oral implantology from India, sent in this contribution for the photo challenge 'SHARP'.

Dr Singh writes: 'This is the skeleton of an alligator showing the jaws and the sharp teeth. The sharp crestal bone can also be appreciated. I took this photo in a museum in a national park in India. The alligator measured six metres in length and has 110 teeth'.

If you would like to send in the view from your practice/workplace or a photo with the theme SHARP, email the News Editor, Kate Quinlan, on k.quinlan@ nature.com.

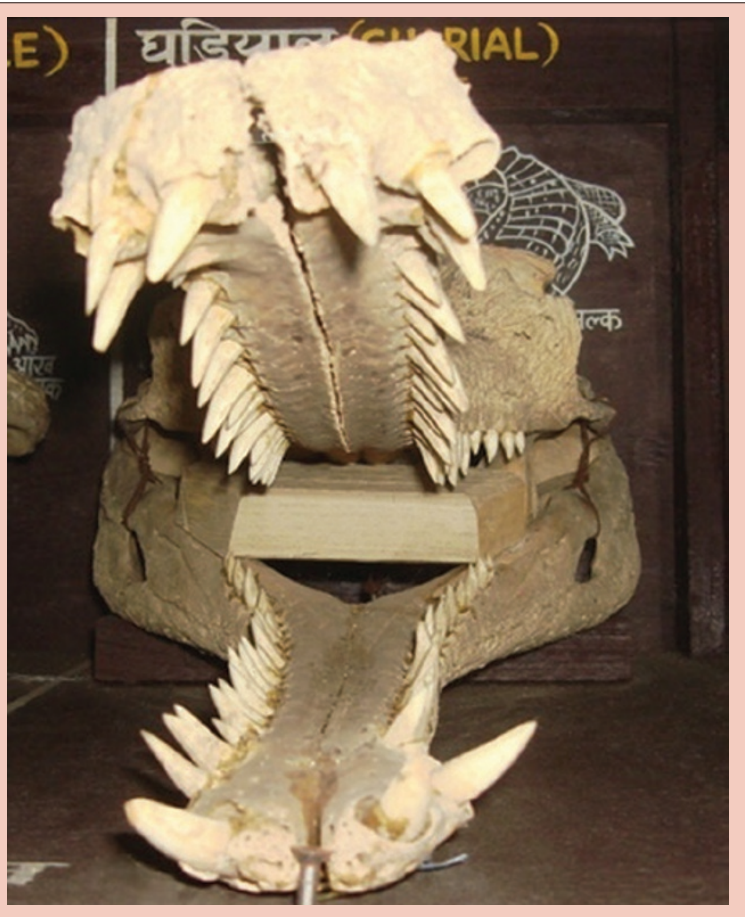

\section{BOOK REVIEW}

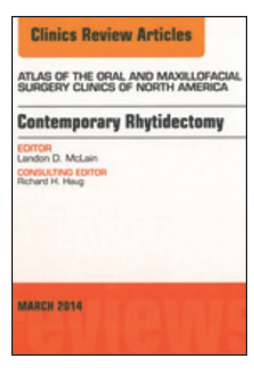

CONTEMPORARY RHYTIDECTOMY, AN ISSUE OF ATLAS OF THE ORAL AND MAXILLOFACIAL SURGERY CLINICS OF NORTH AMERICA

L. D. McLain, R. H. Haug (eds)
Elsevier
price £157.99; pp 102
ISBN 9780323286961

Contemporary rhytidectomy is a 102-page, comprehensive publication by the Atlas of the Oral and Maxillofacial Surgery Clinics of North America. This clinical review article follows from their previous edition, Complications in facial plastic surgery.

Rhytidectomy, or 'facelift' as it is commonly referred to, is the most comprehensive cosmetic facial procedure. The first contemporary face-lifting techniques were being performed in the early 1900s. The scope of practice and technical skills required are highlighted in the book. The articles aim to offer the reader an introduction to the most common rhytidectomy techniques used today, along with complimentary procedures. It is worth noting that the articles in this book are focused on the American oral maxillofacial practitioner, or those with an interest in expanding knowledge in aesthetic maxillofacial surgery.

A wealth of experience is included in the publication with guest editor Landon D. McLain. The nine chapters guide the reader from the initial evaluation of the facelift patient, surgical anatomy, varied types of rhytidectomies and adjunctive techniques, to the final chapter focused on re-operative face and neck lifts. Additionally, there is suggested reading after each chapter to aid the reader.

The case reports in the article 'The opportunistic rhytidectomy' is particularly detailed in providing a complete overview of technique with pre-, sequential surgical and post-operative photographs. The discussion in biplane facelift principles highlights the clinical importance of a thorough understanding of anatomy and surgical limitations on each approach.

I thoroughly enjoyed reading the article on surgical anatomy, which discusses the ageing face and the clinical applications. I feel the book must be praised for its interesting collection of clinical photographs and fine detailed medical illustrations that conceptualise the information on the surgical procedures.

The high quality of these clinical review articles continues to provide insightful clinical perspective reviewing the latest topics in facial surgery, enabling the reader to stay current and improve patient outcomes. Throughout the book, the importance of evidence-based dentistry is emphasised and also, its importance for clinical application. The text includes interesting perspectives and conflicting ideas that allow the reader to engage in critical thinking, and highlights the 'art' of cosmetic surgery, along with stressing its subjective nature. The series continues with their next publication due this autumn on Syndromes of the head and neck.

C. HARRISON 